\title{
Exactly Soluble Diffeomorphism Invariant Theories
}

\author{
Gary T. Horowitz ${ }^{\star}$ \\ Institute for Theoretical Physics, University of California, Santa Barbara, CA 93106, USA
}

\begin{abstract}
A class of diffeomorphism invariant theories is described for which the Hilbert space of quantum states can be explicitly constructed. These theories can be formulated in any dimension and include Witten's solution to $2+1$ dimensional gravity as a special case. Higher dimensional generalizations exist which start with an action similar to the Einstein action in $n$ dimensions. Many of these theories do not involve a spacetime metric and provide examples of topological quantum field theories. One is a version of Yang-Mills theory in which the only quantum states on $S^{3} \times R$ are the $\theta$ vacua. Finally it is shown that the three dimensional Chern-Simons theory (which Witten has shown is intimately connected with knot theory) arises naturally from a four dimensional topological gauge theory.
\end{abstract}

\section{Introduction}

Gravity in three dimensions is often considered trivial since the field equation $R_{\mu \nu}=0$ implies that the spacetime is flat. However this does not mean that there is only one classical solution. On a manifold of topology $\Sigma \times R$, where $\Sigma$ is a compact two manifold of genus $g>1$, there is a $12 g-12$ dimensional space of flat metrics [1]. Since the solution space is finite dimensional, the theory is analogous to ordinary particle mechanics and the quantization should be straightforward.

However if one writes the three dimensional Einstein action in canonical form using the usual canonical variables (the spatial metric and its conjugate momentum) then one finds that the constraints associated with diffeomorphism invariance are

$$
\begin{array}{r}
p_{i j} p^{i j}-\left(p_{i}^{i}\right)^{2}-R=0, \\
D_{i} p^{i j}=0,
\end{array}
$$

where $D_{i}$ and $R$ are the covariant derivative and scalar curvature of the spatial metric. These are essentially identical in form to those of the four dimensional

\footnotetext{
* On leave from the Department of Physics, University of California, Santa Barbara, CA, USA
} 
theory. There is no obvious simplification. To quantize the theory, one must either solve the constraints to isolate the "true degrees of freedom" (reduced phase space approach) or impose the constraints as operator conditions on the wave functions (Dirac approach). From this viewpoint, neither approach seems any easier than the four dimensional case.

In a recent paper [1], Witten presented a different approach to this problem. He showed that the Einstein action in three dimensions is exactly equivalent to a Chern-Simons term for the Poincare group. Using the gauge theory analog, the quantization was straightforward and the theory was shown to be exactly soluble. The fact that the theory was three dimensional was used crucially in at least two different ways. First, the Chern-Simons term is a three form and hence has a well defined integral only over a three dimensional manifold. Second, the Chern-Simons term requires an invariant, nondegenerate metric on the Lie algebra. In most previously studied cases, the Lie group was semisimple and there was a natural metric to use, namely the Cartan-Killing metric. However the Poincare group is not semisimple. It is easy to show [1] that there is an invariant invertible metric only in three dimensions. ${ }^{1}$

One motivation for the present work is to show that the importance of three dimensions is (at least in some respects) an illusion. Three dimensions is certainly crucial for showing the equivalence of gravity and the Poincare Chern-Simons term. While this is a remarkable and surprising result, it is not needed for solving the theory. We will construct a class of field theories in arbitrary dimensions which are all diffeomorphism invariant and exactly soluble. Three dimensional gravity will be included as a special case. These theories share several common features. In each case there is no local dynamics. The solution space is finite dimensional and related to the topology of the underlying manifold. The actions are linear in time derivatives and so are easily adapted to canonical quantization. Since the actions are invariant under diffeomorphisms, there are constraints in the canonical formalism. The theory can be quantized using either the reduced phase space or Dirac procedure and the results are equivalent.

Another motivation is to explore the program initiated by Atiyah [2] and Witten $[3,4]$ to relate results in low dimensional topology to quantum field theory. (See also [5-7].) Some of the theories we will consider have a spacetime metric but no local dynamics (like three dimensional gravity). However most will not involve any spacetime metric at all. These may be viewed as examples of "topological quantum field theories." For instance, we will see that there is a natural quantum field theory description for de Rham cohomology. There is also a formulation of Yang-Mills theory in which there is no local dynamics, but the quantum states include the $\theta$ sectors.

Witten has shown that a three dimensional quantum field theory based on the Chern-Simons action provides a natural framework for studying knot theory [8]. We will see that there is a close connection between this Chern-Simons theory

\footnotetext{
${ }^{1}$ If $P_{a}$ are the generators of translations and $J_{a b}$ are the generators of Lorentz transformations, the required metric is $\varepsilon^{a b c} P_{a} J_{b c}$
} 
and a four dimensional topological gauge theory. For example, on $S^{3} \times R$, the topological gauge theory has a unique quantum state. In canonical quantization this can be expressed as a function on the space of gauge fields on $S^{3}$. We will see that the unique solution to the constraints is $\psi(A)=e^{i g S_{\mathrm{CS}}(A)}$, where $S_{\mathrm{CS}}$ is the Chern-Simons action! ( $g$ is a parameter which will be discussed.) Given a closed curve $C$ on $S^{3}$ one can consider the operator $W(C)=\operatorname{Tr} P e^{\oint c A}$ corresponding to the trace (in some representation) of the Wilson line integral of the gauge field. $W(C)$ is a configuration operator in this theory. If $k$ is an integer, matrix elements of operators of this type between the states $\psi_{g}$ and $\psi_{g+k}$ yields the three dimensional Chern-Simons theory and knot invariants.

The physical motivation for studying topological quantum field theories is that they may describe a phase of unbroken diffeomorphism invariance in quantum gravity $[3,9]$. This is very appealing in light of other arguments [10] that the spacetime metric should be a derived quantity in quantum gravity. In fact it is tempting to carry this idea one step further. As we have mentioned, some topological quantum field theories have a small number of quantum states. This can be viewed as resulting from a very large gauge invariance. One may hope that the fundamental theory has a sufficiently large gauge group that there is just a single quantum state. ${ }^{2}$ This would obviate the need for finding preferred initial conditions or a physical law governing initial conditions [12]. We will discuss some simple examples of theories of this type.

Since canonical quantization will be used extensively, we now briefly review this procedure for systems with constraints. The standard framework (which will be sufficient for most of the theories considered here) is when the phase space $\Gamma$ is the cotangent bundle of a configuration space $X . \Gamma$ has a natural symplectic structure, i.e. an invertible closed two form $\Omega_{A B}$. Classically, constraints are real valued functions $C_{i}$ on $\Gamma$. We will mostly be interested in the case when the constraints are first class, i.e. the Poisson brackets of the constraints are proportional to the constraints. In this case, there are two standard approaches to quantization. The first starts by defining the constraint surface to be the subspace of $\Gamma$ on which $C_{i}=0$. For each constraint, the vector field $\Omega^{A B} \partial_{B} C_{i}$ is tangent to the constraint subspace. The set of integral curves of these vector fields define the reduced phase space. A point in the reduced phase space represents a solution to the constraints modulo gauge freedom. The symplectic structure restricted to the constraint subspace will in general be degenerate, but pulled back to the reduced phase space it is nondegenerate. If the reduced phase space is also a cotangent bundle over a reduced configuration space $X^{\prime}$, then there is a natural representation of quantum states in terms of wave functions on $X^{\prime}$. More generally, one has to introduce a polarization which divides the reduced phase space into "position" and "momentum" variables.

In the second approach, the Dirac approach, one first ignores the constraints

\footnotetext{
${ }^{2}$ The hope that there will be a single quantum state of the universe has been expressed before [11]. Generalizations of topological quantum field theories may offer the first concrete examples of theories of this type
} 
and quantizes the unconstrained system. The constraints $C_{i}$ then become operators acting on wave functions on the full configuration space $X$. The "physical states" are defined to be those annihilated by the constraints.

This paper is organized as follows. In Sect. 2 we begin with the simplest examples of exactly solvable diffeomorphism invariant theories. They are "free field theories" in the sense that the action is quadratic. These theories do not involve a spacetime metric and are closely related to de Rham cohomology. They may be thought of as examples of "quantum cohomology." In Sect. 3 we generalize to nonlinear theories. It is here that we will find a connection between topological gauge theories and knot theory. In Sect. 4 we consider gravity. We will show that Witten's solution to $2+1$ dimensional gravity is simply a special case of one of the theories considered in Sect. 3. Higher dimensional generalizations are also discussed. We conclude in Sect. 5 with a discussion of these results. A possible generalization of some of the knot invariants to higher dimensional surfaces is mentioned.

\section{Quantum Cohomology}

We begin with one of the simplest examples of the theories we wish to consider. Let $M$ be a four dimensional manifold. Let $F_{\mu \nu}$ be a Maxwell field on $M$, and $B_{\mu \nu}$ be a two form "axion" potential whose gauge invariant field strength is $H_{\mu v \rho}=3 \partial_{[\mu} B_{v \rho]}$. The action is simply ${ }^{3}$

$$
S=6 \int_{M} F_{[\mu v} B_{\rho \sigma]} .
$$

Note that this action does not involve a spacetime metric. Since the integrand is a four form, the integral is well defined and invariant under (orientation preserving) diffeomorphisms. It is also invariant under the usual Maxwell gauge transformation and the axion gauge transformation $\delta B_{\mu \nu}=\partial_{[\mu} v_{v]}$ for any one form $v_{v}{ }^{4}$ The field equations obtained by varying with respect to $B_{\mu \nu}$ and the gauge potential $A_{\mu}$ are simply $F_{\mu \nu}=0$ and $H_{\mu v \rho}=0$. Recall that the $p^{\text {th }}$ de Rham cohomology group $H^{p}$ of $M$ is defined to be the space of curl free $p$ forms modulo the curl of a $p-1$ form. Thus the gauge inequivalent classical solutions for $A_{\mu}$ and $B_{\mu \nu}$ are precisely the first and second cohomology groups of $M$. If $M=R^{4}$, these cohomology groups are trivial and all solutions are gauge equivalent to $A_{\mu}=0=B_{\mu v}$. However on a general manifold $M$, there is a finite (nonzero) dimensional space of solutions.

At first sight there appears to be a problem with analysing this theory as an ordinary field theory. In conventional theories, the space of classical solutions is always even dimensional. This is because it can be identified with the phase space of the system which must be even dimensional in order to admit a symplectic

\footnotetext{
${ }^{3}$ As usual, square brackets around $n$ indices denotes the antisymmetrized sum of all $n$ ! permutations multiplied by $1 / n$ !. The factor of 6 is for later convenience. It arises since the wedge product of a $p$ form and a $q$ form is, by definition, $(p+q) ! / p ! q$ ! times the product of the forms with the indices antisymmetrized

${ }^{4}$ Strictly speaking this is true only if $M$ is compact without boundary. If $M$ has a boundary, then one must either add a surface term to (2.1) or require $v_{v}$ to vanish on the boundary
} 
structure. (Roughly speaking, there must be an equal number of $p$ 's and $q$ 's.) However the above theory defined on a general manifold $M$ need not have an even dimensional space of solutions. For example, if $M=S^{3} \times S^{1}$ then $\operatorname{dim} H^{1}=1$ and $\operatorname{dim} H^{2}=0$ so there is only a one parameter family of solutions. However the identification of the space of solutions with the phase space is through a split of the field equations into initial data and evolution equations. This restricts the manifold to be topologically $\Sigma \times R$, where $\Sigma$ is a compact three manifold. In this case, cohomology groups on $M$ reduce to cohomology groups on $\Sigma$. Fortunately, $\operatorname{dim} H^{1}(\Sigma)=\operatorname{dim} H^{2}(\Sigma)$ for every $\Sigma$. This is a simple consequence of Poincaré duality which states that on a compact orientable ${ }^{5} n$ dimensional manifold, the $p^{\text {th }}$ and $(n-p)^{\text {th }}$ cohomology groups are isomorphic. Thus in the case where the canonical formalism can be applied, the solution space is always even dimensional and there is no inconsistency. We now proceed to canonically quantize this theory.

Since the action is linear in time derivatives it is straightforward to cast the theory into canonical form. Let $M=\Sigma \times R$ and let $t$ be a coordinate labelling the different $\Sigma$ surfaces. Let $t^{\mu}$ be any vector satisfying $t^{\mu} \partial_{\mu} t=1$. Since the fields of interest are forms, the appropriate notion of time derivative is the Lie derivative. Using the fact that

$$
\dot{A}_{v} \equiv L_{t} A_{v}=\partial_{v}\left(A_{\mu} t^{\mu}\right)+t^{\mu} F_{\mu v}
$$

the action becomes

$$
S=\int d t \int_{\Sigma} 3 \dot{A}_{[i} B_{j k]}-3 F_{[i j} B_{k] 0}+A_{0} H_{i j k}
$$

where the indices $i, j, k$ denote fields on $\Sigma$. The canonically conjugate variables are thus the spatial components of $A_{\mu}$ and $B_{\mu v}{ }^{6}$ The time components of these fields act as Lagrange multipliers enforcing the constraints $F_{i j}=0$ and $H_{i j k}=0$. To see what these constraints generate, let $\lambda$ and $v_{i}$ be an arbitrary function and one form on $\Sigma$, and set $C_{1}=\int \lambda H_{i j k}$ and $C_{2}=\int 3 v_{[i} F_{j k]}$. Taking the Poisson brackets one obtains

$$
\begin{aligned}
\delta A_{i} & =\left\{C_{1}, A_{i}\right\}=\partial_{i} \lambda, \\
\delta B_{i j} & =\left\{C_{2}, B_{i j}\right\}=\partial_{[i} v_{j]},
\end{aligned}
$$

which are the usual gauge transformations. (Note that the vanishing of $H_{i j k}$ generates the gauge transformation for $A_{i}$ and vice versa.) One can easily verify that these constraints commute.

One might wonder what happened to diffeomorphism invariance which is also a symmetry of our action. It turns out that when the constraints are satisfied, spatial diffeomorphisms are contained in the gauge transformations! To see this, note that under a diffeomorphism generated by a vector field $\xi^{i}$ on $\Sigma$

$$
\delta B_{i j}=L_{\xi} B_{i j}=-2 \partial_{[i}\left(B_{j j k} \xi^{k}\right)+H_{i j k} \xi^{k}
$$

\footnotetext{
${ }^{5}$ We will assume that all manifolds considered here are orientable

${ }^{6}$ To cast (2.3) into more familiar form, one can fix a volume form $\varepsilon_{i j k}$ and define $\varepsilon^{i j k}$ such that $\varepsilon^{i j k} \varepsilon_{i j k}=6$. Then $3 \dot{A}_{[i} B_{j k]}=\dot{A}_{l}^{*} B^{l} \varepsilon_{i j k}$, where ${ }^{*} B^{i}=\frac{1}{2} \varepsilon^{i j k} B_{j k}$ is the dual to $B_{j k}$. It is now clear that the momentum conjugate to $A_{i}$ is ${ }^{*} B^{i}$
} 
The first term is the usual gauge transformation for $B_{i j}$ and the second vanishes when the constraints hold. The result for $A_{i}$ is similar. Thus, on the constraint surface, the constraints generate spatial diffeomorphisms as well as gauge transformations. We will see in Sect. 5 that, in a certain sense, all diffeomorphisms are represented in the canonical theory.

Even though the original phase space is infinite dimensional, the reduced phase space is finite dimensional and is simply $H^{1}(\Sigma) \times H^{2}(\Sigma)$. The induced symplectic structure is simply related to the intersection pairing of these cohomology groups. Since the reduced phase space is a product space, we can take the simplest polarization and have our wave functions be either functions of $A_{i}$ or $B_{i j}$. Thus the Hilbert space of quantum states is simply the space of square integrable functions on $H^{1}(\Sigma)$ (or $H^{2}(\Sigma)$ ). It is worth emphasing that there is no Hamiltonian and no Schroedinger equation for these wave functions to satisfy. The Hamiltonian was constrained to vanish (like all diffeomorphism invariant theories on manifolds with no spatial boundary) and we solved the constraints to obtain the reduced phase space.

If one wants to impose the constraints as operators on the wave functions, one starts by choosing a polarization on the full phase space. In the present case, we can start with arbitrary functions of all $A_{i}$ 's. The constraint $C_{2}$ becomes a configuration operator which acts by multiplication. $C_{2} \psi=0$ says that $\psi$ has support only on curl free $A_{i}$ 's. The constraint $C_{1}$ is linear in the variables conjugate to $A_{i}$, and hence $C_{1} \psi=0$ is a first order differential equation

$$
\int_{\Sigma} \partial_{i} \lambda \frac{\delta \psi}{\delta A_{i}}=0
$$

which is the statement that the wave functions are gauge invariant. So the Hilbert space of states is again arbitrary square integrable functions on $H^{1}(\Sigma)$.

Classical observables in this theory are arbitrary functions on $H^{1}(\Sigma) \times H^{2}(\Sigma)$. A basis for such observables are given by nontrivial one and two dimensional surfaces in $\Sigma$, i.e. elements of the first and second homology groups. For example, for each two surface $S$ one can consider $\int_{S} B_{i j}$. This is clearly gauge invariant and gives a linear map from $H^{2}(\Sigma)$ to the reals. Given a state one can compute expectation values of these observables in the usual way.

This theory can be generalized in many directions. First let $M=\Sigma \times R$, where $\Sigma$ is a compact $n$ dimensional manifold, and consider a $p$ form $A$ and a $n-p$ form $B$ on $M^{7}$. We consider the action

$$
S=\int_{M} d A \wedge B
$$

The above analysis goes through essentially unchanged. The space of classical solutions are the $p^{\text {th }}$ and $(n-p)^{\text {th }}$ cohomology classes of $\Sigma$. The canonical variables are the spatial components of $A$ and $B$, and the constraints are the vanishing of

\footnotetext{
7 For notational convenience, we will drop the indices when discussing higher rank forms. The standard notation $\wedge$ for wedge product and $d$ for exterior derivative (or curl) will be used
} 
the spatial components of the field strengths $H=d B$ and $F=d A$. Quantization can be carried out in either of the two standard approaches and the result is that the quantum states are the square integrable functions on $H^{p}(\Sigma)$ ( or $H^{n-p}(\Sigma)$ ). One can of course consider sums of terms such as (2.8) (with forms of different rank on the same manifold).

Of special interest is the case where $n=2 p+1$ and $p$ is odd. In addition to the theories discussed above with two fields $A$ and $B$, one can now consider a new theory with a single $p$ forms $A$. The action is the abelian Chern-Simon term $S=\int d A \wedge A$. (If $p$ was even, $S$ would be a total derivative.) The space of classical solutions is now just the $p^{\text {th }}$ cohomology group of $M, H^{p}(M)$. Fortunately, the dimension of $H^{p}(\Sigma)$ (for a compact $2 p$ dimensional $\Sigma$ ) is always even, and there is a natural symplectic structure on this space ${ }^{8}$, so it can again be interpreted as a phase space. Since the momentum conjugate to $A$ is $A$ itself, to construct the quantum theory one must divide $A$ into "position" and "momentum" variables. If $\Sigma$ admits a complex structure, then the choice of complex structure leads to a natural choice of polarization. On a complex manifold, the decomposition of the tangent space into holomorphic and antiholomorphic vectors leads to a decomposition of $p$ forms into $(r, p-r)$ forms with $r$ holomorphic and $p-r$ antiholomorphic components. If $p$ is odd, one can consider wave functions which depend on $p$ forms with more holomorphic than antiholomorphic components. For example, in the three dimensional Chern-Simons theory, the wave functions would be functions of $A_{z}$ and not $A_{z}$.

If $M$ has dimension $4 m$ for some integer $m$ and $p=2 m$, then one can consider the following generalization of the above theories:

$$
S=\int_{M} F \wedge B-\frac{1}{2} B \wedge B
$$

where $F=d A$ and $B$ each has rank $2 m$. (If the rank of $B$ is odd, the second term vanishes identically.) $S$ is clearly invariant under $\delta A=d \lambda$ for any $2 m-2$ form $\lambda$. However the second term breaks the $\delta B=d v$ symmetry so one might naively expect there to be more gauge inequivalent solutions. In fact the field equations are now $F-B=0$ and $d B=0$. The second equation is a consequence of the first so one can pick any curl free $F$ and set $B=F$ to obtain a solution. However (2.9) is actually invariant under a much larger gauge invariance

$$
\begin{aligned}
& \delta A=w, \\
& \delta B=d w,
\end{aligned}
$$

where $w$ is an arbitrary form of rank $2 m-1$. One can use this invariance to locally set $A$ to zero. The gauge inequivalent solutions are thus characterized by a rank $2 m$ form $F$ which is curl free, modulo the curl of a $2 m-1$ form, i.e. $H^{2 m}(M)$. Now for the first time we encounter a topological obstruction to canonical quantization. The problem is that even if $M=\Sigma \times R, H^{2 m}(M)=H^{2 m}(\Sigma)$ need not be even dimensional. This breakdown of the canonical formalism seems to be related to

${ }^{8}$ If $A^{1}$ and $A^{2}$ are two elements of $H^{p}(\Sigma)$, the symplectic structure is simply $\int_{\Sigma} A^{1} \wedge A^{2}$ 
the fact that if $H^{2 m}(\Sigma) \neq 0$, then $A$ is not globally defined. For now we consider only the case $H^{2 m}(\Sigma)=0$.

As one might hope, even if one had failed to notice the enlarged symmetry (2.10), the canonical formalism includes it automatically. The canonical variables are again the spatial components of $B$ and $A$. The only change from the canonical analysis of (2.8) is that the constraint $C=\int_{\Sigma} w \wedge F$ (where $w$ is an arbitrary $2 m-1$ form $)$ is replaced by $\tilde{C}=\int_{\Sigma} w \wedge(F-B)$. This generates precisely the gauge transformations (2.10) on the canonical variables.

Since $H^{2 m}(\Sigma)=0$, the reduced phase space is just a point. But it is of interest to see what happens if one imposes the constraints as operators. On the full phase space we consider wave functions $\psi(A)$ on the space of all $2 m-1$ forms $A$ on $\Sigma$. The constraint $d B=0$ implies that $\psi$ is invariant under changing $A$ by the curl of a $2 m-2$ form. The constraint $\widetilde{C} \psi=0$ yields a surprise. The differential equation is

$$
\frac{1}{i} \int_{\Sigma} w \frac{\delta \psi}{\delta A}=\left(\int_{\Sigma} w \wedge F\right) \psi .
$$

The unique solution (up to an overall factor) is $\psi=e^{i S_{\mathrm{Cs}}}$, where $S_{\mathrm{CS}}=\int A \wedge d A$ is the abelian Chern-Simons action! Since the reduced phase space is a single point, one expects there to be only one quantum state. But the fact that the Chern-Simons action arises in this way is somewhat surprising. We will see an analogous result for nonabelian gauge theories in the next section.

\section{Nonabelian Generalizations}

The theories we have discussed so far are perhaps the simplest examples of topological quantum field theories. They are all "free field theories" in the sense that the action is quadratic in the basic fields. It is therefore perhaps not surprising that they could be quantized so easily. We now show that this linear structure is not essential by generalizing to a class of "interacting field theories." For definiteness we again start with a four dimensional manifold $M$. Let $G$ be any Lie group. Let $A_{\mu}^{a}$ be a gauge field for $G$ on $M$ (i.e. a connection on a principle $G$ bundle over $M)$. Let $B_{\mu v a}$ be a two form taking values in the dual to the Lie algebra of $G$ which we will denote $L^{*}$. Consider the action

$$
S=6 \int_{M} F_{[\mu \nu}^{a} B_{\rho \sigma] a}
$$

where $F_{\mu \nu}^{a}=2 \partial_{[\mu} A_{v]}^{a}+\left[A_{\mu}, A_{v}\right]^{a}$ is the usual Yang-Mills field strength. As in our previous examples, no spacetime metric is needed to define this theory. Note that no metric on the Lie algebra is needed either, so the group $G$ need not be semisimple. The field equations obtained by extreming with respect to $B_{\mu v a}$ and $A_{\mu}^{a}$ are $F_{\mu \nu}^{a}=0$, and $D_{[\mu} B_{\rho \sigma] a}=0$, where $D_{\mu}=\partial_{\mu}+\left[A_{\mu}, \quad\right]$ is the gauge covariant derivative ${ }^{9}$. The

\footnotetext{
9 Actually, this is the expression for $D_{\mu}$ acting on a Lie algebra valued field. If the Lie algebra admits an invariant metric, there is no need to distinguish between the Lie algebra and its dual. If not, then one defines $D_{\mu} v_{b}=\partial_{\mu} v_{b}-v_{m} C_{a b}^{m} A_{\mu}^{a}$, where $C_{a b}^{m}$ are the structure constants of the group and $v_{b}$ is an arbitrary field taking values $L^{*}$
} 
first states that $A_{\mu}^{a}$ is a flat connection, and the second states that the covariant curl of $B_{\mu v a}$ vanishes.

In addition to diffeomorphisms, $S$ is invariant under both the usual nonabelian gauge transformations and also under

$$
\delta B_{\mu v a}=D_{[\mu} v_{v] a}
$$

where $v_{v a}$ is one form valued in $L^{*}$. (After integrating by parts, $\delta S$ vanishes by the Bianchi identity for $F_{\mu v}^{a}$.) If one fixes the connection, the field equation and gauge transformations for $B_{\mu v a}$ are linear. However for an arbitrary $A_{\mu}^{a}$ the transformation $\delta B_{\mu v a}=D_{[\mu} v_{v] a}$ does not leave the field equation invariant since applying the covariant curl twice does not yield zero but rather $F_{\mu \nu}^{a}$. When $A_{\mu}^{a}$ satisfies its field equation, $F_{\mu \nu}^{a}=0$, and one can use $D_{\mu}$ to define cohomology classes of forms with values in $L^{*}$. Thus the space of gauge inequivalent solutions consists of the gauge inequivalent flat connections together with elements of the second cohomology group with values in $L^{*}$. We will see below that if $M=\Sigma \times R$, this space has the structure of a cotangent bundle and hence is ideally suited for canonical quantization.

Since the action (3.1) is linear in time derivatives, it is again easy to cast into canonical form. Let $M=\Sigma \times R$. The only fields with time derivatives are the spatial components of $A_{\mu}^{a}$. The conjugate momenta are the spatial components of $B_{\mu v a}$ and the time components again act as Lagrange multipliers. The action becomes ${ }^{10}$

$$
S=\int d t 3 \int_{\Sigma} \dot{A}_{[i}^{a} B_{j k] a}-F_{[i j}^{a} B_{k] 0 a}+A_{0}^{a} D_{[i} B_{j k] a}
$$

The constraints are

$$
F_{i j}^{a}=0, \quad D_{[i} B_{j k] a}=0 .
$$

To see what these constraints generate, let $\tau^{a}$ and $v_{i a}$ be arbitrary fields on $\Sigma$ and set

$$
\begin{aligned}
& C_{1}=\int_{\Sigma} 3 \tau^{a} D_{[i} B_{j k] a}, \\
& C_{2}=\int_{\Sigma} 3 F_{[i j}^{a} v_{k] a} .
\end{aligned}
$$

The Poisson brackets yield

$$
\delta A_{i}^{a}=D_{i} \tau^{a}, \quad \delta B_{i j a}=D_{[i} v_{j] a}+\left[B_{i j}, \tau\right]_{a} .
$$

Thus the constraint $C_{1}$ generates the usual nonabelian gauge transformations for $A_{i}^{a}$ and $B_{i j a}$ and the constraint $C_{2}$ generates the gauge transformation (3.2) for $B_{i j a}$. Just as in the abelian case, spatial diffeomorphisms are again included in the gauge transformations when the constraints are satisfied. To see this, let $\xi^{i}$ be a vector on $\Sigma$. Then

$$
L_{\xi} A_{j}^{a}=\partial_{j}\left(A_{i}^{a} \xi^{i}\right)+2 \xi^{i} \partial_{[i} A_{j]}^{a}=D_{j}\left(A_{i}^{a} \xi^{i}\right)+\xi^{i} F_{i j}^{a}
$$

10 The dot denotes Lie derivative as before. The Lie derivative of forms taking values in a vector space can be defined just as for ordinary forms. This is because the Lie derivative is local so one can choose a basis in the vector space and reduce it to a collection of ordinary forms 
The first term is the usual gauge transformation with the gauge parameter $\tau^{a}=A_{i}^{a} \xi^{i}$, and the second is proportional to the constraint.

From Eqs. (3.4) and (3.6) one sees that the reduced phase space consists of the space $\mathcal{N}$ of gauge inequivalent flat connections on $\Sigma$ together with elements of the second cohomology class with values in $L^{*}$. This space is precisely the cotangent bundle of $\mathscr{N}$. To see this, consider first the cotangent bundle of the space of all connections. The fiber over $A_{i}^{a}$ is the set of all linear maps from infinitesimal connections to the reals. This is just the space of all $B_{i j a}$ via the map $\int_{\Sigma} \delta A_{[i}^{a} B_{j k] a}$. In passing to the reduced phase space, we first require that $A_{i}^{a}$ is flat. This implies that $B_{i j a}$ and $B_{i j a}+D_{[i} v_{j] a}$ (for any $v_{j a}$ ) give the same linear map and must be identified. Next we require that two $A_{i}^{a}$ 's are equivalent if they are related by gauge transformations. In order for $B_{i j a}$ to have the same action on gauge equivalent $A_{i}^{a}$ 's it must satisfy $D_{[i} B_{j k] a}=0$. These are precisely the field equations and gauge transformations for $B_{i j a}$. Since the reduced phase space is a cotangent bundle, there is a natural symplectic structure and a natural polarization defined by the fibers. The quantum Hilbert space consists of square integrable functions on the space of gauge inequivalent flat connections $\mathcal{N}$.

Alternatively, one can quantize using the Dirac procedure. One starts with functions of all connections $\psi\left(A_{i}^{a}\right)$. The constraint $C_{2} \psi=0$ implies that $\psi$ has support only on flat connections and the constraint $C_{1} \psi=0$ implies that $\psi$ is invariant under gauge transformations. So one again obtains the same quantum Hilbert space.

Classically, gauge invariant observables in this theory are again functions on the reduced phase space. One class of configuration observables are the Wilson lines (holonomy) of the gauge field around noncontractible loops $\operatorname{Tr} P e^{\oint A}$. It turns out that one cannot obtain a gauge invariant object by integrating $B_{i j a}$ over a two dimensional surface since the path ordering is not well defined [13].

There is a subtlety in the above discussion which we now pause to explain. We have seen that the reduced phase space is the cotangent bundle of the space $\mathscr{N}$ of gauge inequivalent flat connections on $\Sigma$. In most cases $\mathscr{N}$ is disconnected for two distinct reasons. Gauge inequivalent flat connections are characterized by their holonomy around non-contractible loops. This is equivalent to specifying homomorphisms from $\pi_{1}(\Sigma)$ into $G$ (up to conjugation by $G$ ). The space of such homomorphisms is in general disconnected. For the particular case $\Sigma=S^{3}$, the first homotopy group is trivial so one might expect the reduced phase space to consist of a single point. However there is a second consideration which must be taken into account. The constraints generate infinitesimal gauge transformations. When one takes the quotient to obtain the reduced phase space, one is only including gauge transformations which are connected to the identity. For most manifolds and most gauge groups, there are large gauge transformations which cannot be continuously connected to the identity. In these cases the reduced phase space becomes disconnected. The number of disjoint pieces is just the number of connected components of the space of maps from $\Sigma$ into $G$. For $\Sigma=S^{3}$ this is $\pi_{3}(G)$ which for every simple Lie group is the integers $Z$. Thus rather than a single point, the reduced phase space becomes a countably infinite number of points which can be 
labeled by the winding number $k$. The quantum Hilbert space consists of complex valued sequences which are square summable. Since one can view these sequences as Fourier coefficients for functions on a circle, a natural basis is

$$
\psi_{\theta}(k)=e^{i k \theta} .
$$

These are just the usual $\theta$ states of Yang-Mills theory. (Under a large gauge transformation which maps $k$ to $k+1, \psi_{\theta}$ goes to $e^{i \theta} \psi_{\theta}$.) Thus on $S^{3} \times R$, the theory described by (3.1) can be viewed as a form of Yang-Mills theory in which all local dynamics has been removed and only the $\theta$ states remain.

If $\Sigma$ is a homology sphere, i.e. $H^{1}(\Sigma)=0$, then the set of gauge inequivalent flat connections is discrete. The number of such connections counted with appropriate signs, yields Casson's invariant [14] which is analogous to the Euler number for Floer cohomology [15]. Witten has constructed a topological gauge theory in which the quantum states are precisely the Floer cohomology groups [3]. His theory is certainly not equivalent to the one considered here since e.g. each flat connection in the theory (3.1) is accompanied by the corresponding $\theta$ sectors.

One can again generalize the theory described by (3.1) to $n$ dimensional manifolds. However unlike the abelian case, where both $A$ and $B$ could become higher rank forms, in this case the nonlinearity of the action in $A$ (and its geometric interpretation as a connection) requires that $A$ must remain a Lie algebra valued one form. Thus the $n$ dimensional generalization consists of a gauge field $A$ and a $n-2$ form $B$ taking values in the dual to the Lie algebra. The canonical quantization is essentially unchanged. The Hilbert space is square integrable functions on the space of gauge inequivalent flat connections on a $n-1$ dimensional manifold $\Sigma$.

In three dimensions, $B$ is a one form and (assuming there is a metric on the Lie algebra) one might be tempted to identify $B$ with $A$. However this does not lead to a gauge invariant action. Under the usual Yang-Mills gauge transformation, $B$ transforms covariantly and $A$ does not. However one can of course modify this action so that it is gauge invariant,

$$
S_{\mathrm{CS}}=\int \operatorname{Tr}\left(A \wedge F-\frac{1}{3} A \wedge A \wedge A\right),
$$

where $\operatorname{Tr}$ denotes contraction with the Lie algebra metric. This is just the Chern-Simons action. The entire reduced phase space is now just the space of gauge inequivalent flat connections. If $G$ is compact, this space is also compact since (as we have mentioned) the connections can be characterized by their holonomy which takes values in $G$. This is a significant difference with the theories we have discussed above. In the case of (3.1), if $G$ is compact, then $\mathscr{N}$ is compact, but the reduced phase space is the cotangent bundle of $\mathcal{N}$ which is always noncompact. Canonical quantization of the Chern-Simons action requires extra care since a polarization cannot be defined on a general compact manifold. This has been analysed in detail by Witten [8].

If $M$ is four dimensional and there is a metric on the Lie algebra of $G$, one can consider the following action: 


$$
S=6 \int_{M} F_{[\mu \nu}^{a} B_{\rho \sigma] a}-\frac{1}{2 g} B_{[\mu \nu}^{a} B_{\rho \sigma] a} .
$$

This is the nonabelian generalization of the theory (2.9) discussed at the end of Sect. 2, with a parameter $g$ added. The field equations are $g F_{\mu \nu}^{a}-B_{\mu \nu}^{a}=0$ and $D_{[\mu} B_{\rho \sigma] a}=0$. The second equation is clearly a consequence of the first. The action is invariant under diffeomorphisms, the usual nonabelian gauge transformations, and the nonabelian generalization of $(2.10)$

$$
\delta A_{\mu}^{a}=w_{\mu}^{a}, \quad \delta B_{\mu \nu}^{a}=g D_{[\mu} w_{v]}^{a},
$$

where $w_{\mu}^{a}$ is a Lie algebra valued one form. (Note that even though $F_{\mu \nu}^{a}$ need not be zero, there is no contradiction between the gauge transformation $\delta B_{\mu \nu}^{a}=g D_{[\mu} w_{\nu]}^{a}$ and the field equation $D_{[\mu} B_{\rho \sigma]}^{a}=0$ because the gauge transformation now also changes $A_{\mu}^{a}$.) This gauge invariance allows one to locally set $A_{\mu}^{a}$ to zero. More precisely, given a principle $G$ bundle over $M$, any two connections in this bundle can be related by a guage transformation of the above type. Since $B_{\mu \nu}^{a}$ is uniquely determined by $F_{\mu \nu}^{a}$, there is precisely one classical solution for each principle $G$ bundle over $M$.

This action is similar to the gauge invariant actions for Witten's topological gauge theory. If one substitutes the solution $B_{\mu \nu}^{a}=g F_{\mu \nu}^{a}$ into (3.10) one obtains $\int \operatorname{Tr} F \wedge F$ which is the gauge invariant action proposed [6] for Witten's theory. In addition, the gauge transformation (3.11) is precisely the one used to reproduce Witten's theory. An alternative action proposed by Labastida and Pernici [7] used a two from $B_{\mu \nu}^{a}$ which is very similar to the one above.

As usual, in the canonical form of this theory, the constraints are the spatial components of the field equations. Thus one constraint is identical to $C_{1}$ given in (3.5) and the other is

$$
\tilde{C}_{2}=\int_{\Sigma} 3\left(g F_{[i j}^{a}-B_{[i j}^{a}\right) w_{k] a} .
$$

One can easily verify that they generate the spatial components of the gauge transformations. The reduced phase space thus consists of a collection of points, one for each $G$ bundle over $\Sigma$. Note the difference with the theory (3.1). In the previous case, large gauge transformations resulted in different points in the reduced phase space. In the present theory, this is not the case. The enlarged gauge transformations (3.11) now connect the different winding numbers. For example, consider $\Sigma=S^{3}$. Principle $G$ bundles over $S^{3}$ are characterized by $\pi_{2}(G)$. But this is trivial for every $G$. So the only bundle is $S^{3} \times G$. Thus the reduced phase space now consists of a single point rather than a countably infinite number of points. The theta sectors have disappeared.

Of particular interest is the result of Dirac quantization. As before, the requirement that $C_{1} \psi_{g}(A)=0$ implies that $\psi_{g}$ is invariant under the usual Yang Mills gauge transformation. The requirement $\tilde{C}_{2} \psi_{g}(A)=0$ yields a first order differential equation whose unique solution (up to an overall constant) is

$$
\psi_{g}(A)=e^{i g S_{\mathrm{CS}}(A)},
$$

where $S_{\mathrm{CS}}$ is the three dimensional Chern-Simons action! One can clearly choose 
the overall constant independently for each topologically distinct $G$ bundle over $\Sigma$. Thus, if there are $N$ different bundles, the Hilbert space is $N$ dimensional.

The form of the solution to the constraints (3.13) has a particularly striking consequence if one views different values of $g$ as labelling different sectors of the same theory rather than different theories. To motivate this, first note that in the abelian theory (2.9), if one had added an analogous parameter $g$ it would have no effect, since it would be absorbed by a simple rescaling $B \rightarrow B / g^{1 / 2}$ and $A \rightarrow g^{1 / 2} A$. In the nonabelian theory one of course cannot remove $g$ in this way. Also note that $g$ does not take values on a circle, but is an arbitrary real parameter. Indeed, the wave functions $\psi_{g}$ are not invariant under $g$ and go to $g+s$ for any constant $s$. In Yang-Mills theory, one often considers different values of the theta angle as labelling different sectors of the same theory rather than different theories. This is largely a matter of choice since the different theta sectors are completely independent. There are no gauge invariant operators which relate one value of $\theta$ to another. A similar feature holds if $g$ and $g^{\prime}$ do not differ by an integer. This can be seen by computing the inner product between the two states $\psi_{g}$ and $\psi_{g^{\prime}}$. The inner product involves an integral over all gauge fields which can be divided into an integral over sectors differing by large gauge transformations ${ }^{11}$. Under a large gauge transformation, the Chern-Simons action changes by $2 \pi$ times an integer. If we denote the integral over one sector by $V$, then the inner product is

$$
\left\langle\psi_{g^{\prime}} \mid \psi_{g}\right\rangle=\int D A e^{i\left(g-g^{\prime}\right) S_{\mathrm{CS}}}=\sum_{n} e^{2 \pi i n\left(g-g^{\prime}\right)} V=\delta\left(g-g^{\prime}\right) V .
$$

However, if $g-g^{\prime}$ is an integer, then the inner product is nonzero and the sectors can communicate.

For each closed curve $C$ in $\Sigma$, consider the operator $W(C)=\operatorname{Tr} P e^{\oint_{C} A}$. This is a configuration operator in the theory (3.10) which acts by multiplication. The matrix elements of products of such operators between the states $\psi_{g}$ and $\psi_{g+k}$ ( $k$ integer) is

$$
\left\langle\psi_{g}\left|\prod_{i} W\left(C_{i}\right)\right| \psi_{g+k}\right\rangle=\int D A \prod_{i} W\left(C_{i}\right) e^{i k S_{\mathbf{c s}}}
$$

The right-hand side can be viewed as a functional integral in a three dimensional quantum theory described by the Chern-Simons action. This is precisely the starting point for Witten's recent discussion of the Jones polynomial for knot theory! One of the main motivations for that work was to provide a three dimensional description for topological invariants characterizing knots. We have seen that the Chern-Simons theory that Witten starts with arises naturally from a four dimensional topological quantum field theory.

\section{Gravity}

We now consider the special case of the action (3.1) when the manifold $M$ is three dimensional and $G$ is the Lorentz group $S O(2,1)$. The action becomes

${ }^{11}$ Since the space of connections is an affine space, these sectors are not disconnected. One can define the $n^{\text {th }}$ sector to consist of connections for which $2 \pi n<S_{\mathrm{CS}}<2 \pi(n+1)$ 


$$
S=3 \int R_{[\mu \nu}^{a b} B_{\rho] a b},
$$

where we have replaced $F$ by the more suggestive variable $R$ and expressed the Lie algebra in terms of the fundamental representation of antisymmetric tensors on a three dimensional space. $R_{\mu \nu}^{a b}$ is the curvature of a Lorentz connection $\omega_{\mu}^{a b}$ and $B_{\rho a b}$ is a one form with values in the dual to the Lorentz Lie algebra. But $B_{\mu a b}$ is equivalent to a triad of vectors through $e_{\mu}^{a}=\varepsilon^{a b c} B_{\mu b c}$. It now becomes clear that (4.1) is precisely the Einstein action in first order form. Note how the spacetime metric enters this theory. The general theories we have considered until now do not involve a spacetime metric. Three dimensional gravity does. The difference is that since $B_{\mu a b}$ is equivalent to a triad of vectors, if they are linearly independent, one can define a metric such that $e_{\mu}^{a}$ is an orthonormal basis.

Let us apply the general canonical quantization of the previous section to this theory. As always, let $M=\Sigma \times R$, where $\Sigma$ is a compact two manifold. The canonical variables are the spatial components of $e_{\mu}^{a}$ and $\omega_{\mu}^{a b}$ which are subject to the constraints

$$
R_{i j}^{a b}=0, \quad T_{i j}^{a} \equiv 2 \partial_{[i} e_{j]}^{a}+2 \omega_{[i}^{a b} e_{j] b}=0 .
$$

The reduced phase space is the space $\mathcal{N}$ of gauge inequivalent flat $\operatorname{SO}(2,1)$ connections on $\Sigma$ together with elements of the first cohomology class taking values in the dual to the $S O(2,1)$ Lie algebra. From the general argument of the previous section, we know that this space is just the cotangent bundle to $\mathscr{N}$. The quantum Hilbert space thus consists of square integrable functions on $\mathscr{N}$.

This is precisely the result obtained by Witten in his solution to $2+1$ dimensional gravity. Although the mathematics is the same, his interpretation was completely different. Witten interpreted $(e, \omega)$ as a connection for the Poincaré group. With the appropriate choice of metric on the Lie algebra, the action (4.1) is the Chern-Simons term for this connection. The constraints (4.2) imply that the curvature of this connection vanishes. The reduced phase space is thus the space of gauge inequivalent flat Poincare connections. Witten notes that this space is the cotangent bundle to the space of flat Lorentz connections $\mathcal{N}$. Thus the Hilbert space is again the space of square integrable functions on $\mathscr{N}$.

Note that there is no difficulty in quantizing this theory using the Dirac approach. In direct analogy with theories in the previous section, if one starts with wave functions on the space of all connections $\psi(\omega)$, imposing the constraint that $R_{i j}^{a b}$ annihilate $\psi$ says $\psi$ has support on flat connections and requiring $T_{i}^{a b}$ annihilate $\psi$ implies that $\psi$ is invariant under gauge transformations. So the result is the same Hilbert space as before.

As we have discussed, the space of gauge inequivalent flat connections is disconnected for two reasons. The first is due to different classes of homomorphisms from $\pi_{1}(\Sigma)$ into $G$. Witten argues that only one of these classes should be included corresponding to the one for which spacetimes contain only "tame" singularities. The second reason is that the constraints only generate gauge transformations connected to the identity. Large gauge transformations correspond to homotopy classes of maps from $\Sigma$ into $S O(2,1)$. Since $S O(2,1)$ is topologically $S^{1} \times R^{2}$, if $\Sigma=S^{2}$ there are no large gauge transformations. However, for all other Riemann 
surfaces there are. These are the analogs of the $\theta$ sectors for three dimensional gravity.

We have seen two different interpretations of the constraint (4.2b). One is that the covariant curl of the one form $e_{i}^{a}$ vanishes and defines certain cohomology groups. The other is that part of the curvature of the Poincaré connection vanishes. Actually, the most common interpretation of $(4.2 \mathrm{~b})$ is neither of these. It is just that the connection is torsion free. This will be important when we now generalize to higher dimensions.

We will consider the four dimensional case with a manifold of the form $\Sigma \times R$. The generalization to higher dimensions is straightforward. Let $e_{\mu}^{a}$ be a basis of one forms and $\omega_{\mu}^{a b}$ be a Lorentz connection. The Einstein action, again in first order form, is ${ }^{12}$

$$
S=6 \int R_{[\mu \nu}^{a b} e_{\rho}^{c} e_{\sigma]}^{d} \varepsilon_{a b c d} .
$$

In the usual approach, one takes $e_{\mu}^{a}$ and $\omega_{\mu}^{a b}$ as independent variables. The variation with respect to $\omega_{\mu}^{a b}$ implies that the connection must be torsion free, and the variation with respect to $e_{\mu}^{a}$ yields Einstein's equation. This action is again in a form linear in time derivatives and hence ideally suited for canonical quantization. Only the spatial components of $\omega_{\mu}^{a b}$ enter with time derivatives. However $\omega_{i}^{a b}$ and $e_{i}^{a}$ are clearly not canonically conjugate variables in more than three dimensions. The momentum conjugate to $\omega_{i}^{a b}$ is

$$
p_{i j a b}=e_{[i}^{c} e_{j]}^{d} \varepsilon_{a b c d} .
$$

So the higher dimensional Einstein action is subject to an extra constraint that $p_{i j a b}$ can be expressed in terms of a collection of one forms $e_{i}^{a}$ as in (4.4).

For the moment, let us drop this extra constraint and quantize the theory

$$
S=6 \int R_{[\mu \nu}^{a b} p_{\rho \sigma] a b} .
$$

But this is precisely of our general form (3.1) with $G=S O(3,1)$. In particular, one of the constraints says that the full $S O(3,1)$ curvature (restricted to $\Sigma$ ) must vanish. This may seem puzzling since we have dropped a constraint and are thus considering a theory which is less restrictive than Einstein's. Yet we have obtained a constraint which says that the full curvature must vanish rather than the Ricci curvature. The resolution is that we have ignored the torsion. Flat Lorentz connections do not correspond to flat spacetimes. Even on $R^{n}$, there is an infinite number of spacetimes with flat connections. In fact, given any metric, one can pick an orthonormal basis at each point and define a connection by requiring that its components vanish in this particular frame. The result is a flat metric compatible connection. But of course the torsion is in general nonzero. These connections are called teleparallel [17]. So in this sense, the theory (4.5) includes all spacetimes as solutions. However, in terms of the canonical variables $(\omega, p)$, the space of solutions is finite dimensional (as in all the theories considered here). Canonical quantization proceeds as before, and the Hilbert space is square integrable functions on gauge inequivalent flat $S O(3,1)$ connections.

12 This is often called the Einstein-Cartan theory. For a review of its canonical quantization see [16] 
Of course general relativity in four dimensions is not an exactly soluble theory (as far as we know). Nevertheless, it is intriguing that it can be expressed in a form which is closely related to one that is. To make this connection more explicit, we start with the action (4.5) in canonical form

$$
S=3 \int d t \int_{\Sigma} \dot{\omega}_{[i}^{a b} p_{j k] a b}-R_{[i j}^{a b} p_{k] 0 a b}+\omega_{0}^{a b} D_{[i} p_{j k] a b} .
$$

One must now pick a tetrad $e_{\mu}^{a}$, and a Lagrange multiplier $\lambda_{i}^{a b}$. If one adds to (4.6) the constraint

$$
C=\int_{\Sigma} \lambda_{[i}^{a b}\left(p_{j k] a b}-e_{j}^{c} e_{k]}^{d} \varepsilon_{a b c d}\right)
$$

and restricts the Lagrange multiplier $p_{k 0 a b}$ to be of the form $e_{k}^{c} e_{0}^{d} \varepsilon_{a b c d}$, the theory is equivalent to general relativity. Note that in this approach $e_{i}^{a}$ is not a canonical variable. Its dynamics is determined as follows. The constraint (4.7) does not commute with the other constraints. (These are second class constraints.) The requirement that they be preserved in time is

$$
\dot{C} \equiv\{C, H\}+\frac{\partial C}{\partial t}=0,
$$

where $H$ is the canonical Hamiltonian constructed from (4.6) and (4.7). This determines the evolution of $e_{i}^{a}$ (and $\lambda_{i}^{a b}$ ). Note that our previous theories have only had commuting (first class) constraints. These are always preserved under evolution and the (unrestricted) Lagrange multipliers are the generators of gauge transformations.

Although solutions to the theory (4.5) do not correspond to flat spacetimes, one can easily quantize a theory which is based on flat spacetimes. This can be achieved by considering our previous action (3.1) with $G=$ Poincare group. $^{13}$ Decomposing in terms of the Lorentz and translation parts the action becomes

$$
S=6 \int R_{[\mu \nu}^{a b} p_{\rho \sigma] a b}+T_{[\mu \nu}^{a} M_{\rho \sigma] a},
$$

where $T_{\mu \nu}^{a}$ is the torsion defined in terms of the tetrad $e_{\mu}^{a}$ (the translation part of the connection) in analogy to (4.2b). The field equations now require the connection to be both flat and torsion free. Hence these are flat spacetimes in the usual sense. In addition there are extra degrees of freedom associated with the cohomology classes of $p_{\mu v a b}$ and $M_{\mu v a}$. The canonical variables are the spatial components of both the connection and the tetrad as well as their conjugate momentum. The reduced phase space is the cotangent bundle to the space $\mathcal{N}^{\prime}$ of gauge inequivalent flat torsion free connections restricted to $\Sigma$. The quantum states are square integrable functions on $\mathscr{N}^{\prime}$.

The above considerations can easily be generalized to any dimension, but in four dimensions it is of particular interest to add a cosmological constant term. The Einstein action is now

$$
S=6 \int\left(R_{[\mu \nu}^{a b} e_{\rho}^{c} e_{\sigma]}^{d}-2 \Lambda e_{\mu}^{a} e_{v}^{b} e_{\rho}^{c} e_{\sigma}^{d}\right) \varepsilon_{a b c d} .
$$

13 Recall that no metric is needed on the Lie algebra to construct this theory 
The momentum conjugate to $\omega_{i}^{a b}$ is again given by (4.4). If we drop this constraint as before, then the action becomes

$$
S=6 \int R_{[\mu \nu}^{a b} p_{\rho \sigma] a b}-\frac{\Lambda}{2} p_{[\mu \nu}^{a b} p_{\rho \sigma]}^{c d} \varepsilon_{a b c d} .
$$

This is just a special case of the theory (3.10) discussed at the end of Sect. 3 with $G=S O(3,1)$. The cosmological constant $\Lambda$ plays the role of the parameter $g$. Note that we are not using the Cartan-Killing metric for $S O(3,1)$. In any dimension, given two Lorentz generators $L^{a b}$ and $M^{a b}$, one has the inner product $L^{a b} M^{c d} g_{a c} g_{b d}$. In four dimensions, there is a second invariant, invertible metric given by $L^{a b} M^{c d} \varepsilon_{a b c d}$. It is clearly this metric which is being used in (4.11). Since the previous discussion held for any Lie algebra metric, the canonical quantization proceeds as before. Using the Dirac approach, one finds a unique quantum state which is $\psi(\omega)=e^{i S_{\mathrm{cs} /} A}$, where $S_{\mathrm{CS}}$ is the Lorentz Chern-Simons action (with the above Lie algebra metric). The theory (4.11) may be related to recent discussions of topological gravity [18].

\section{Discussion}

In this section we will discuss some implications and speculations concerning the above theories. The first is related to the choice of canonical variables for general relativity. In Sect. 1 it was pointed out that in terms of the usual spatial metric and conjugate momentum, the constraint equations of three dimensional gravity are no simpler than the four dimensional case. As we have discussed, the key observation which allows one to quantize three dimensional general relativity is not that it is equivalent to the Chern-Simons term for the Poincare group, but rather that one should take the basic canonical variables to be the full Lorentz connection restricted to a spacelike surface. It is clear why the use of the Lorentz connection simplifies the canonical approach. The spatial metric and extrinsic curvature are highly dependent on the embedding of the spatial surface into the spacetime. Even in flat spacetime there are "wiggly" surfaces for which these quantities do not take a simple form. The constraints associated with these variables must be complicated enough to admit all of these possibilities. The connection is less dependent on the choice of surface and the constraints simplify. This is the key idea behind Ashtekar's new variables for four dimensional general relativity [19]. He uses the self dual part of the Lorentz connection as the fundamental canonical variable. This choice of variables arises naturally from an action which is a chiral version of (4.3) [20]. Although the full space of solutions to the constraints is not yet understood, some solutions have been found which, interestingly enough, are closely connected to knot theory [21].

How do the theories described here compare with previously studied diffeomorphism invariant theories? In most previous discussions of theories with a dynamical metric, one introduces a lapse function $N$ and a shift vector $N^{i}$ to describe the surfaces used to set up the canonical theory [22]. However in the absence of a spacetime metric, this is not possible. Indeed, $N$ and $N^{i}$ represent certain components of the spacetime metric. Another way to see this is that in the 
absence of a metric, one cannot decompose the vector $t^{\mu}$ (connecting one surface to the next) into normal and tangential components.

However topological quantum field theories are still invariant under changing ones choice of surfaces. To see this, one can use the following observation ${ }^{14}$. Given any action $S(\alpha, \beta)$ depending on two fields $\alpha$ and $\beta$, one has an invariance

$$
\delta \alpha=\varepsilon \delta S / \delta \beta, \quad \delta \beta=-\varepsilon \delta S / \delta \alpha .
$$

This is clearly trivial when the field equations are satisfied. In fact one can show that (5.1) is the most general invariance which reduces to the identity on shell. Since this invariance is present even in theories with no local gauge invariance, it is not associated with constraints. Two symmetries which differ by (5.1) are effectively equivalent.

Now consider for simplicity the action (2.1), $S=\int F \wedge B$ in four dimensions. The general invariance (5.1) applied to this action yields

$$
\delta A_{\mu}=\xi^{v} F_{v \mu}, \quad \delta B_{\mu \nu}=\xi^{\rho} H_{\rho \mu \nu}
$$

for an arbitrary vector field $\xi^{\mu}$. Under a diffeomorphism

$$
\delta A_{\mu}=\partial_{\mu}\left(\xi^{v} A_{v}\right)+\xi^{v} F_{v \mu},
$$

and similarly for $B_{\mu v}$. So modulo the transformation (5.2) this is precisely a gauge transformation. In the canonical formalism, we have seen that the constraints generate the spatial components of the gauge transformations. The transformation properties of the Lagrange multipliers are obtained by demanding that the action be invariant under the transformations generated by the constraints. This recovers the time components of the gauge transformations. Thus the full gauge invariance is included in the canonical theory, and hence the full diffeomorphism invariance as well. Although we have illustrated this for the theory (2.1), similar results hold for all theories discussed here.

Other finite dimensional models for general relativity have been considered. The most well known are perhaps the minisuperspace models [23]. In the usual canonical approach to quantum gravity, one considers wave functions on the space of all three metrics. By imposing symmetries, one can reduce the space of all three metrics to a smaller space called minisuperspace. A standard choice is to consider only homogeneous spacetimes. In this case the classical field equations reduce to ordinary differential equations which have a finite dimensional space of solutions. (The dimension of this space depends on the amount of anisotropy and number of matter fields one wishes to include.) However, even with this restriction, the constraints cannot usually be solved exactly (neither classically nor quantum mechanically) and one is forced to resort to semiclassical approximations. Although one reason for this is undoubtedly that these models include nontrivial dynamics, part of the complication may be removed by using a new choice of canonical variables. Another significant difference is that for minisuperspace models, the finite dimensionality arises from an ad-hoc restriction on the fields rather than from constraints derived from the theory.

${ }^{14}$ I thank Mark Henneaux and Claudio Teitelboim for explaining this to me 
The theories described in the previous sections have only been quantized using canonical quantization. The next step is clearly to consider functional integrals. The partition function for many of these theories has been studied by Schwarz [24]. Other functional integral expressions may be related to topological invariants. For example, given an $n$ dimensional manifold $M$, and submanifolds $U, V$ of dimension $p$ and $n-p-1$ respectively, one could compute

$$
\int D A D B\left(\int_{U} A\right)\left(\int_{V} B\right) e^{i S},
$$

where the integral is over all $p$ forms $A$ and $n-p-1$ forms $B$ and $S=\int d A \wedge B$. The result will in general depend on the boundary conditions chosen for the functional integral. However, if $M$ is compact without boundary, the result should be a topological invariant related to the surfaces $U$ and $V$. This may yield information about the linking of $p$ and $n-p-1$ dimensional surfaces in the $n$ dimensional manifold $M$. More general expressions including a product of operators of the above type for a collection of surfaces (or perhaps exponential of such operators) might be related to other topological invariants of these surfaces. Similar expressions can be constructed for the other theories described earlier. In the analogous calculation for the three dimensional Chern-Simons theory [8], an important role was played by the fact that if one splits $M$ into two appropriate pieces, the Hilbert space associated with the boundary is one dimensional. We have seen that this is also true for the theories discussed here. (For example, in the theory described by (2.8) this holds whenever $H^{p}(\Sigma)=H^{n-p-1}(\Sigma)=0$.) So some of the techniques given in [8] may be applicable to evaluate expressions such as (5.4).

It has often been argued that a fundamental theory of quantum gravity will not involve a spacetime metric. Topological quantum field theories are interesting models of theories of this type. They show that a theory without a spacetime metric can be formulated in a well defined way and the states are topological. These theories are too simple to show how a metric might arise "dynamically." String theory may be different. Classically it can be formulated without a spacetime metric [25]. There is a natural solution which does not introduce any metric. Quantizing about this solution can be viewed as constructing an infinite dimensional topological quantum field theory. Since classically there are other solutions with a nonzero spacetime metric, one may hope that the full quantum theory will show a transition from a state with no metric to the approximate classical spacetime we see today.

One of the characteristic features of topological quantum field theories is that they have a very large gauge group. The solution space is reduced from an infinite dimensional space to a finite dimensional one. In fact we have seen examples where there is so much gauge invariance that there is a unique quantum state (up to overall scale). It should be noted that a "miracle" is not required to find a theory for which the quantum state is uniquely determined. To illustrate this, consider an arbitrary scalar field theory described by a (Euclidean) action $S(\phi)$ on $n$ dimensional flat space. We will construct an $n+1$ dimensional theory for which the only quantum state is $\psi(\phi)=e^{i S(\phi)}$. We start with the action 


$$
I=\int d t \dot{S}=\int d t \int d^{n} x F(\phi) \dot{\phi},
$$

where $F(\phi)=\delta S / \delta \phi$. The momentum $p$ conjugate to $\phi$ is clearly $F(\phi)$. So the action, in canonical form, is

$$
I=\int p \dot{\phi}-\lambda(p-F),
$$

where $\lambda$ is a Lagrange multiplier which enforces the constraint $p=F$. Classically this constraint generates the gauge transformations $\delta \phi=f, \delta p=-f \delta F / \delta \phi$, where $f$ is an arbitrary function. So all values of $\phi$ can be connected by these gauge transformations. Using the Dirac procedure, quantum states must satisfy

$$
\frac{1}{i} \int f \frac{\delta \psi}{\delta \phi}=\left(\int f F(\phi)\right) \psi \text {. }
$$

The unique solution is $\psi(\phi)=e^{i S(\phi) 15}$.

It is very appealing to imagine that the ultimate theory of the universe will have such a large gauge group that there is only one state. A metric and local dynamics might arise as some form of "symmetry breaking". This would seem to be an attractive alternative to finding natural boundary conditions for the wave function of the universe. In a theory of this type, no boundary conditions would be needed.

Acknowledgements. It is a pleasure to thank Jonathan Halliwell, Jim Hartle, Joe Polchinski, Andy Strominger, Karen Uhlenbeck, and Don Witt for discussions. This work was supported in part by the Alfred P. Sloan Foundation and the National Science Foundation under Grants PHY85-06686 and PHY82-17853, supplemented by funds from NASA.

Note Added. It has recently been shown that expression (5.4) does yield the linking number of the $p$ and $n-p-1$ dimensional surfaces $U$ and $V$. See G. Horowitz and M. Srednicki,: "A Quantum Field Theoretic Description of Linking Numbers and Their Generalization," Santa Barbara preprint UCSB-TH-89-14; and M. Blau and G. Thompson,: "Topological Gauge Theories of Antisymmetric Tensor Fields," Trieste preprint SISSA 39/89/FM

\section{References}

1. Witten, E.: Nucl. Phys. B311, 46 (1988)

2. Atiyah, M.: New invariants of three and four dimensional manifolds, to appear in the Symposium on the Mathematical Heritage of Hermann Weyl. Wells, R. et. al., (eds.). (Univ. of North Carolina, May 1987)

3. Witten, E.: Commun. Math. Phys. 117, 353 (1988)

4. Witten, E.: Commun. Math. Phys. 118, 411 (1988)

5. Baulieu, L., Grossman, B.: Phys. Lett. B212, 351 (1988); Phys. Lett. B214, 223 (1988); Yamron, J.: Phys. Lett. B213, 325 (1988)

6. Baulieu, L., Singer, I.: Topological Yang-Mills Symmetry, to appear in the proceedings of Conformal

${ }^{15}$ From a functional integral viewpoint, the fact that there is a unique quantum state follows immediately from the fact that since the action (5.5) is a total derivative, the amplitude for a given history is independent of the history and depends only on the endpoints. I thank Steve Martin for pointing this out 
Field Theory and Related Topics (Annecy, France, March 1988); Brooks, R., Montano, D., Sonnenschein, J.: Phys. Lett. B214, 91 (1988)

7. Labastida, J., Pernici, M.: Phys. Lett. B212, 56 (1988)

8. Witten, E.: Commun. Math. Phys. 121, 351-399 (1989)

9. Tseytlin, A.: J. Math. Phys. 15, L105 (1982)

10. See e.g. Penrose, R.: In Magic without magic. Klauder, J. (ed.). San Franciscó Freeman 1972

11. See e.g. De Witt, B.: Phys. Rev. 160, 1113 (1967)

12. Hartle, J., Hawking, S.: Phys. Rev. D28, 2960 (1983); Hartle, J.: In: $13^{\text {th }}$ Texas Symposium on Relativistic Astrophysics. Ulmer M. (ed.). Singapore: World Scientific 1987; Vilenkin, A.: Phys. Rev. D33, 3560 (1986)

13. Teitelboim, C.: Phys. Lett. B167, 63 (1986)

14. Taubes, C.: Casson's Invariant and Gauge Theory. Harvard Univ. preprint (1988)

15. Floer, A.: Commun. Math. Phys. 118, 215 (1988)

16. Isenberg, J., Nester, J.: In: General relativity and gravitation Vol. 1, Held, A. (ed.). New York: Plenum Press 1980; Hehl, F., von der Heyde, P., Kerlick, G., Nester, J.: Rev. Mod. Phys. 48, 393 (1976)

17. Hayashi, N., Shirafuji, T.: Phys. Rev. D19, 3524 (1979)

18. Witten, E.: Phys. Lett. B206, 601 (1988); Labastida, J., Pernici, M.: Phys. Lett. B213, 319 (1988)

19. Ashtekar, A.: Phys. Rev. Lett. 57, 2244 (1986); Phys. Rev. D36, 1587 (1987); New Perspectives in Canonical Gravity. Naples: Bibliopolis 1988

20. Samuel, J.: Pramana 28, L429 (1987); Jacobson, T., Smolin, L.: Phys. Lett. B196, 39 (1987); Class. Quantum Grav. 5, 583 (1988)

21. Rovelli, C., Smolin, L.: Loop space representation of quantum gravity. Rome preprint; Phys. Rev. Lett. 61, 1155 (1988)

22. For a review see Kuchar, K.: In: Quantum gravity 2. Isham, C., Penrose, R., Sciama, D. (eds.). Oxford: Oxford University Press 1981

23. For a reviews, see Hartle, J.: In: High energy physics. Bowick, M., Gursey, F. (eds.). Singapore: World Scientific 1985; Misner, C.: In: Magic without Magic. Klauder, J. (ed.). San Francisco: Freeman 1972

24. Schwarz, A.: Lett. Math. Phys. 2, 247 (1978)

25. Horowitz, G., Lykken, J., Rohm, R., Strominger, A.: Phys. Rev. Lett. 57, 283 (1986); Hata, H., Itoh, K., Kugo, K., Kunitomo, H., Ogawa, K.: Phys. Lett. B175, 138 (1986)

Communicated by S.-T. Yau

Received December 9, 1988; in revised form May 12, 1989 
